\title{
Aplicación de sensores remotos para el análisis de cobertura vegetal y cuerpos de agua
}

\author{
Application of remote sensors for the analysis of vegetation cover and water bodies \\ Jaris Veneros ${ }^{1,4 *}$, Ligia García ${ }^{1}$, Eli Morales ${ }^{1,4}$, Víctor Gómez ${ }^{1}$, \\ Mariana Torres ${ }^{2}$, Fernando López-Morales ${ }^{3}$
}

\begin{abstract}
RESUMEN
Este trabajo evalúa la utilidad de sensores remotos para el análisis de cobertura vegetal y cuerpos de agua en estudios de conservación y medio ambiente. La investigación tiene como objetivos determinar las aplicaciones de imágenes satelitales en estudios de cobertura y los usos de los VANT (Vehículos Aéreos No Tripulados) en estudios ambientales. La determinación de las aplicaciones de imágenes satelitales y de los VANT se realizó mediante la revisión de artículos científicos, tesis, libros y resúmenes en congresos. Se encontraron un total de veinte aplicaciones para análisis de cobertura y cuerpos de agua empleando imágenes satelitales y VANT. Para análisis ambientales mediante imágenes satelitales se reportaron diez estudios: cobertura boscosa, expansión urbana, índices de vegetación, cambio de coberturas vegetales, deforestación, distribución espacial del agua, monitoreo del agua, dinámica lagunar, parámetros de calidad de agua y los umbrales del Índice de Nieve de Diferencia Normalizada. Para análisis ambientales mediante los VANT se reportaron diez estudios: abundancia vegetal, dinámica de la población de plantas, conservación ecológica, vegetación acuática, mapeo de vegetación, calidad del agua, dinámica fluvial, flujo de ríos, mapas batimétricos de un lago y variaciones vegetales acuáticas. Se concluye que los principios físicos de la percepción remota explican, a través de leyes, el funcionamiento de los sensores para brindar información satelital, como imágenes satelitales que brindan información con una resolución menor a 10 metros, aplicadas a estudios de áreas de mayor extensión $(\mathrm{km})$. En áreas pequeñas, para obtener información real y precisa, se usa un Vehículo Aéreo No Tripulado (VANT), equipado con una cámara multiespectral que brinda información con una resolución mayor a $10 \mathrm{~cm}$. Por lo tanto, es necesario conocer las limitaciones, ventajas y diferencias de estos dos sistemas, con el fin de planificar investigaciones que utilicen esta información y contribuyan a la protección y conservación de áreas afectadas por elementos naturales y antrópicos.
\end{abstract}

Palabras clave: cámaras multiespectrales, imagen satelital, teledetección, vehículos aéreos no tripulados.

\begin{abstract}
This work analyzes remote sensors' usefulness to analyze vegetation cover and water bodies in conservation and environmental studies. This research aims to determine satellite images' applications in coverage studies and to ascertain UAV uses (Unmanned Aerial Vehicles) in environmental studies. The determination of the applications of satellite images and the UAV was made by reviewing scientific articles, theses, books, and abstracts at conferences. A total of twenty applications were found for coverage and water body studies using satellite images and UAV. For environmental studies using satellite images, ten studies were reported. These are forest cover, urban expansion, vegetation indices, vegetation cover change, deforestation, the spatial distribution of water, water monitoring, lagoon dynamics, water quality parameters, and the Normalized Difference Snow Index thresholds. Otherwise, for environmental studies using the UAV, ten studies were reported. These are plant abundance, plant population dynamics, ecological conservation, aquatic vegetation, vegetation mapping, water quality, fluvial dynamics, river flow, bathymetric maps of a lake, and aquatic plant variations. It is concluded that the physical principles of remote perception explain through laws the operation of sensors to provide satellite information, such as satellite images that provide information with a resolution less than 10 meters, applied to studies of areas of significant extension (Km). For small areas, an Unmanned Aerial Vehicle (UAV) is used to obtain real and accurate information, which is implemented with a multispectral camera to provide information with a resolution greater than $10 \mathrm{~cm}$. Therefore, it is necessary to know the limitations, advantages, and differences of these two systems to plan investigations that use this information and contribute to the protection and conservation of areas affected by natural and anthropogenic elements.

Keywords: multispectral cameras, Satellite imagery, remote sensing, unmanned aerial vehicles.
\end{abstract}

1 Instituto de Investigación, Innovación y Desarrollo para el Sector Agrario y Agroindustrial de la Región Amazonas, Universidad Nacional Toribio Rodríguez de Mendoza de Amazonas, Chachapoyas, Perú.

2 Fondo Nacional de Desarrollo Científico, Tecnológico y de Innovación Tecnológica, Perú.

3 Colegio de Postgraduados - Campus Montecillo, México.

4 Instituto de Investigación para el Desarrollo Sustentable de Ceja de Selva (INDES-CES), Universidad Nacional Toribio Rodríguez de Mendoza de Amazona. Chachapoyas, Perú.

* Autor para correspondencia (jaris.veneros@untrm.edu.pe)

Fecha de Recepción: 2 de Junio, 2020.

Fecha de Aceptación: 15 de Septiembre, 2020. 


\section{Introducción}

En la actualidad existen grandes problemas ambientales, como el calentamiento global y la crisis hídrica, que ocasionan principalmente impactos adversos en nuestros recursos naturales. Para contrarrestar esta situación, es necesaria la preservación de ecosistemas, como los bosques, que son importantes en la captación, conservación, purificación y reposición del agua (MontielGonzález et al., 2015). Asimismo, los bosques absorben y fijan $\mathrm{CO}_{2}$, regulando el clima global. Por lo tanto, a medida que el clima cambia, el recurso hídrico también lo hace, causando un aumento significativo en el deshielo de glaciares, el nivel del mar, las sequías y lluvias torrenciales que afectan el ecosistema global. De igual manera, los océanos alteran la modulación de los niveles de $\mathrm{CO}_{2}$, variando la temperatura global y el impacto en transformación de nutrientes para especies marinas. Por consiguiente, el conocimiento obtenido a partir de las investigaciones científicas es imprescindible para continuar con la conservación de ecosistemas y mejorar la calidad de vida humana (FAO, 2016).

Tiria et al. (2018) señalan que la zona geográfica de la Amazonía es más vulnerable al cambio climático, porque existe alta presión sobre los ecosistemas que amenaza su estabilidad, por actividades antrópicas, principalmente la deforestación. El fenómeno de la deforestación causa déficit hídrico, baja retención y alteración en la humedad que a su vez cambia la cobertura y el uso de suelo, incentivando la alteración de radiación. El Perú registró en 2016 un total de 164.662 ha deforestadas de bosques húmedos amazónicos. Esta cifra es 5,2\% mayor que la de 2015. Los bosques de esta zona geográfica representan el $94 \%$ del país. Hubo una pérdida total de 1.974.208 208 ha de bosques húmedos amazónicos durante los años 2001 y 2016, lo que se tradujo en un promedio anual de 123388 ha (MINAM, 2017).

En la región Amazonas la cobertura vegetal y los cuerpos de agua enfrentan una severa disminución, situación preocupante y riesgosa para el Área de Conservación Privada (ACP) Tilacancha, donde se encuentran las fuentes de agua que alimentan a la ciudad de Chachapoyas, que actualmente está siendo amenazada por deforestación, incendios y otras actividades antrópicas. La información brindada anteriormente no es precisa, por lo que el uso y la aplicación de nuevas tecnologías han permitido potenciar las investigaciones y la gestión de la cobertura vegetal. Cada año se ha obtenido información más real y de alta precisión con el uso de plataformas satelitales, por lo que se ha observado que la cobertura vegetal está aumentando en países como China, Rusia y el norte de Australia, pese a la pérdida de la biodiversidad vegetal que se ha producido en países de América del Sur y el Sureste de Asia (Salas et al., 2018).

En este trabajo de revisión, buscamos dar un panorama amplio de los avances que se han logrado con la aplicación de sensores remotos, para el análisis de cobertura vegetal y cuerpos de agua que contribuyan a su conservación y protección de las Áreas de Conservación Natural (ACN).

\section{Metodología}

\section{Principios físicos de la teledetección}

La teledetección es un conjunto de técnicas que nos ayudan a obtener información a distancia de un determinado objeto situado sobre la superficie terrestre. Esta información va más allá del alcance del ojo humano, y es obtenida mediante la interacción de los principales elementos: sensor, objeto de estudio y flujo energético, este último también denominado Espectro Electromagnético. Cuando se analiza el comportamiento espectral de la vegetación y el comportamiento espectral de la cobertura forestal, observamos que la reflectividad se ve afectada por factores como la geometría y posición de las hojas, ángulo de observación, lignina y el tipo de suelo (Martínez-Barbáchano y Solís-Miranda, 2018). En cuanto al comportamiento espectral del agua, la reflectividad que presenta es muy baja, desciende a medida que aumenta la longitud de onda, y puede ser afectada por factores como la clorofila, material en suspensión y profundidad.

\section{Sensores remotos}

Los sensores remotos son sistemas o instrumentos utilizados para recolectar información rápida y viable de un objeto, área o fenómeno a distancia. Dicha información puede ser procesada e interpretada mediante el uso de Sistemas de Información Geográfica (SIG) para tener una mejor comprensión. Los sensores remotos 
instalados a bordo de una plataforma son los más utilizados en los últimos años, y registran la energía electromagnética emitida o reflejada por la superficie de un objeto en distintas bandas del espectro electromagnético. Estos registros incluyen longitudes de onda desde las más cortas (gamma y radiográficas) hasta las más largas (microondas y de radiodifusión). Dentro de las longitudes de onda más cortas están las ultravioleta, que son muy prácticas para los sensores (Herrera et al., 2018).

\section{Clasificación de los sensores remotos}

Los sensores remotos se pueden clasificar en sensores de tipo pasivo y sensores de tipo activo, de acuerdo con la energía que utilizan para obtener la información. Los sensores pasivos dependen de la energía ambiental de una fuente externa y permiten medir la magnitud de la radiación electromagnética reflejada por la superficie terrestre y atmosférica a partir del sol en el espectro visible. El Sensor Infrarrojo Térmico (TIRS por sus siglas en inglés) es un ejemplo de este tipo de sensor, el cual mide la temperatura de la superficie terrestre en dos bandas térmicas con una nueva tecnología que aplica la física cuántica para detectar el calor. Mientras que los sensores activos generan su propia fuente de energía, y son capaces de emitir ondas en la región del espectro de las microondas y recibir la señal que rebota de la superficie terrestre. El sensor de Detección y Localización de Imágenes por Láser (LIDAR por sus siglas en inglés) y el sensor de Detección y Localización por Radio (RADAR por sus siglas en inglés) son sensores activos (Herrera et al., 2018).

\section{Plataformas de teledetección}

Las plataformas son los satélites (LANDSAT, METEOSAT, SPOT, entre otros) o aviones que transportan los equipos requeridos para la captura, almacenamiento y transmisión a distancia de las imágenes, denominados sensores. Existen tres tipos de plataformas de acuerdo con la distancia que tienen de la superficie terrestre: nivel superficial, aéreos, satelitales. Los datos adquiridos de estos satélites son las denominadas imágenes satelitales, la cuales se pueden aplicar a diversos estudios relacionados con la cobertura de la tierra, tipos de vegetación, cuerpos de agua, temperatura de la superficie, temperatura del aire, etc. Las imágenes multiespectrales se pueden utilizar en estudios de cobertura vegetal. Por ejemplo, para determinar la producción de biomasa, cálculo de los índices de vegetación, plagas de la vegetación, monitoreo de bosques, alcance de incendios y minería. Asimismo, para estudios de recursos hídricos como la dinámica de cuerpos de agua, estrés hídrico, presencia de agentes contaminantes y turbidez. Para investigaciones en suelos como tipos de suelos, nivel de humedad, y también pueden ayudar a distinguir rocas superficiales. Igualmente, para estudios de la atmósfera, donde se puede analizar la calidad del aire y contaminantes (Martínez-Barbáchano y Solís-Miranda, 2018).

\section{Imágenes hiperespectrales}

La evolución espectroscópica de imágenes por los años 1980 permitió la creación de los dispositivos llamados hiperespectrales (unión de tecnologías para formar imágenes y la espectroscopia). Dicho término tuvo su origen en el Laboratorio de Propulsión a Chorro de la NASA en California, EE.UU, donde se desarrolló un instrumento hiperespectral llamado espectrómetro de imágenes aéreas/infrarrojas en el aire (AVIRIS por sus siglas en inglés). Las imágenes hiperespectrales son imágenes tomadas por un sensor espectral que mide la reflectancia en más de 200 bandas, y las firmas espectrales más finas o continuas que requieren funciones continuas y derivables. Permiten detectar características y diferencias muy sutiles entre los rasgos de la superficie especialmente en lo que se refiere a vegetación, suelo y rocas. La diferencia entre una imagen multiespectral y una imagen hiperespectral radica fundamentalmente en el número y ancho de las bandas (Ibarrola-Ulzurrun et al., 2017).

\section{Procesamiento y análisis de imágenes satelitales}

Las imágenes satelitales descargadas tienen una serie de deficiencias sobre su aspecto, y para corregirlas se deben realizar diversos procedimientos a fin de conseguir una mejor interpretación de la información. Estas imágenes incluyen mejoras en el ajuste del contraste y en la aplicación de color, mediante correcciones geométricas, radiométricas y atmosféricas. También mejoran el contraste al aumentar la calidad visual de la imagen e incluyen aquellas técnicas de 
expansión y comprensión de su histograma para adaptarlo al dispositivo de salida (Liu et al., 2018).

- La corrección radiométrica es el proceso en el cual se restablecen los valores de la imagen y se depura cualquier anomalía en la radiancia del objetivo alterada por la atmósfera o por los defectos del sensor.

- La corrección geométrica es llamada también georreferenciación. Tiene como finalidad adaptar la imagen a un sistema de coordenadas y a una determinada proyección cartográfica. Las distorsiones pueden ser originadas por movimientos en la plataforma, cambios en la altitud de plataforma, rotación de la tierra y/o relieve del terreno.

- En cambio, la corrección atmosférica tiene la finalidad de eliminar el efecto de los aerosoles y la radiancia intrínseca que se ve reflejado en la imagen.

Los procedimientos de clasificación de imágenes satelitales tienen el objetivo de categorizar automáticamente todos los píxeles en una o diversas clases temáticas, utilizando la reflectancia de cada píxel en diferentes longitudes de onda y un criterio estadístico para asignarlo a una clase espectral correspondiente a un tipo de cobertura de terreno. La información categorizada es usada para generar mapas temáticos. Los métodos de clasificación comúnmente son de dos tipos, de acuerdo con la manera en la que se obtienen las estadísticas de las categorías definidas durante la fase de entrenamiento: métodos supervisados y métodos no supervisados (Álvarez-Rogel y Conesa, 2018).

- Clasificación supervisada. Es necesario contar con áreas de entrenamiento para este proceso, construyendo a partir de estas una identificación numérica con la que se compara cada píxel de la imagen. Las áreas de entrenamiento ayudarán a analizar el grado de discriminación y el grado de error de esta clasificación.

- Clasificación no supervisada. Se realiza cuando no se cuenta con áreas de entrenamiento y se desarrolla a través del análisis clúster. Este es un proceso que busca agrupar un conjunto de datos observados en un número determinado de grupos o clústeres de acuerdo con su tendencia natural y una comparación en campo. El investigador no interviene en el proceso de clasificación y no existen áreas de entrenamiento. Pero los resultados son poco exactos.

\section{Vehículos aéreos no tripulados}

Los Vehículos Aéreos No Tripulados (VANT) son avances tecnológicos que permiten obtener ortofotografías y modelos digitales de la topografía de un terreno con una alta resolución, que se utilizan para los estudios geológicos y el desarrollo de trabajos científicos. Son nuevas tecnologías usadas para capturar imágenes multiespectrales de alta resolución, las cuales se ajustan a las condiciones del lugar de estudio como horas del día óptimas para realizar los sobrevuelos, identificación de obstáculos, altura del vuelo, número de fotos por punto, delimitación del área (Fernández-Lozano y Gutiérrez-Alonso, 2016).

La clasificación de los vehículos aéreos no tripulados se da a partir de diferentes aspectos como el uso militar y civil. Por el tipo de control que utilizan: autónomo, monitorizado, supervisado, pre-programado y controlado remotamente. Por la forma que presentan: multirrotores, híbridos y ala fija. Todos están en constantes mejoras tecnológicas para superar sus desventajas al momento de su aplicación en diferentes campos de estudio. Los VANT suelen contar con sistemas de geolocalización por satélite como el Sistema de Posicionamiento Global (GPS por sus siglas en inglés), lo que permite conocer su posición y controlar la estabilidad del aparato. Un aspecto importante de los VANT son los sensores utilizados, de los cuales se pueden capturar imágenes de alta resolución (Pino, 2019).

Las cámaras permiten la captura de imágenes a través de la energía electromagnética terrestre, brindan imágenes de alta resolución, con una información digital georreferenciada y en tiempo real, donde los sensores son las cámaras fotográficas RGB, cámaras térmicas o infrarrojas, cámaras hiperespectrales y multiespectrales (en función de que reproduzcan el espectro de forma continua o discontinua, respectivamente), que proporcionan información digital (Fernández-Lozano y GutiérrezAlonso, 2016).

El plan de vuelo es la ruta o trayectoria de forma automática, que debe seguir un VANT, para la obtención de la información objeto de estudio. Los VANT en su mayoría, dependiendo de la investigación que realicen, pueden ser operados a 
través de un control remoto (manual) o de forma autónoma por medio de un plan de vuelo establecido. Un buen resultado de una investigación se obtiene cuando la operación del VANT es mediante una planificación de vuelo. Además, es primordial tener en cuenta el terreno que se fotografiará (área de estudio), el tipo de cámara que montaremos en el VANT, la hora y día para realizar el vuelo, la escala fotográfica, número de fotogramas, tiempo de vuelo, altura de vuelo y zona de aterrizajedespegue (Duffy et al., 2018).

\section{Aplicación de imágenes satelitales en cobertura vegetal}

Con el interés de conocer la dinámica de una región de excepcional diversidad biológica (Gran Chaco), que contempla cuatro países: Argentina, Bolivia, Paraguay y Brasil, se monitorearon los cambios de cobertura boscosa del Gran Chaco en el año 2013, con el uso de imágenes satelitales de Landsat5 TM, Landsat7 ETM, Landsat8 LCM. Se detectaron cambios de uso de suelo (de bosques a áreas de cultivo y ganadera) en 502.308 ha, equivalente a una tasa de deforestación de 1,376 ha/día (Liu et al., 2018).

En Bogotá se desarrollaron lineamientos metodológicos mediante el uso de herramientas geográficas y teledetección para la detección de cambios, modelamiento y proyección de la expansión urbana y el cambio de coberturas vegetales. Se usaron imágenes satelitales y fotografías aéreas en cuatro periodos de tiempo: 1985, 1995, 2005, 2015, y los resultados mostraron que la cobertura de árboles y arbustos disminuyó en un 56\% (Achicanoy et al., 2018). De acuerdo con Paz (2018), México evaluó la cobertura aérea de la vegetación herbácea, donde se validó la información a través de sensores remotos que permitieron múltiples experimentos de vegetación herbácea, estableciéndose su robustez y estabilidad para ser usados en términos operativos.

Mientras que Liu et al. (2018) monitorearon la evolución espacial de la cobertura vegetal durante 16 años en Tongren, China. Utilizaron imágenes del Landsat7 ETM en 2000 y Landsat8 en 2016, como fuentes de datos que usan un método de sustitución parcial de fase de tiempo diferente para eliminar los efectos de nubes y el modelo de píxeles mediante la Diferencia Normalizada del Índice de Vegetación (NVDI por sus siglas en inglés).
En Perú, con el objetivo de evaluar la expansión urbana y las modificaciones de la cobertura vegetal en el distrito de Végueta-Lima, usaron imágenes satelitales de Landsat7 de los años 1986, 2001, 2005 y 2010 , procesadas en el programa LeoWorks V. 3 para la combinación de bandas, para su posterior análisis visual y análisis de los histogramas. Concluyeron que el incremento de la expansión urbana aumentó de $0.9 \%$ a $7,7 \%$ para los años 1986-2014 y para cobertura disminuyeron desde $18 \%$ hasta $8,2 \%$ para el mismo periodo (Loayza et al., 2017).

En el ACP Tilacancha, Salas et al. (2018) determinaron la deforestación en el área de conservación, por su importancia como una zona de recarga hídrica y de abastecimiento de agua para Chachapoyas. Se aplicó el algoritmo de clasificación supervisada de máxima probabilidad incorporada en el QGIS utilizando datos satelitales multiespectrales obtenidos de Landsat 5 y 8 para los años 1984, 2000 y 2016. Los resultados indican que la cobertura forestal para el primer y segundo periodo se redujo en un $21,63 \%$ y $41,39 \%$, mientras que el área de pajonal se incrementó en un 13,63\% y $18.06 \%$ respectivamente.

\section{Aplicación de imágenes satelitales en la dinámica de cuerpos de agua}

Los cuerpos de agua como lagos, ríos y embalses frescos y salinos cubren solo una pequeña parte de la superficie terrestre, pero son fundamentales para los ecosistemas y para la humanidad. La distribución espacial del agua y sus cambios originan problemas económicos, ambientales y sociales. Feng et al. (2016), para obtener información de los cuerpos de aguas continentales durante el año 2000, adquirieron datos satelitales con $30 \mathrm{~m}$ de resolución (Landsat), índices multiespectrales de agua, vegetación y métricas de terreno. Este conjunto de datos identificó 3.650 .723 $\mathrm{km}^{2}$ de aguas continentales a nivel mundial, de los cuales el 40,65\% corresponde a América del Norte, Asia $(32,77 \%)$, Europa $(9,64 \%)$, África $(8,47 \%)$, América del Sur $(6,91 \%)$ y Oceanía $(1,57 \%)$.

El estudio de cuerpos de agua se ha convertido en un aspecto muy importante, ya que el monitoreo del agua juega un papel importante en la gestión de los recursos hídricos. Kaplan y Avdan (2017) utilizaron un método que combina un índice basado en píxeles (clasificación supervisada y no 
supervisada) y un método basado en objetos en una imagen de satélite Sentinel-2 con una resolución de $10 \mathrm{~m}$ para el estudio de cuerpos de agua (área) presentes en una región montañosa y urbana, ubicados en Macedonia. Concluyeron que con estas técnicas los datos son más exactos comparados con datos de campo y se pueden usar para zonas montañosas u otros tipos de áreas dificultosas.

Se han realizado, además, investigaciones como las de Aliaga et al. (2016), quienes usaron imágenes satelitales de distintas resoluciones espaciales para interpretar la dinámica lagunar en la Región Pampeana argentina. Se utilizó el Índice Estandarizado de Precipitación (IEP) para la determinación de años húmedos, secos y normales. Este análisis mostró que las lagunas pampeanas no se comportan de la misma manera ante los eventos pluviométricos. Su origen, utilización socioeconómica y régimen pluviométrico condicionan su variación espacio-temporal y morfométrica.

En Chile, Briceño et al. (2018), con el uso de la teledetección (imágenes satelitales del sensor OLI de Landsat8), realizaron el estudio de la distribución espacial y la dinámica de los parámetros de calidad de agua del lago Vichuquén-Chile durante el año 2016. Hicieron recolecciones de muestras de campo y mediciones in situ, análisis en laboratorios y procesamientos de imágenes satelitales. Concluyeron que los procedimientos sirvieron para conocer la variabilidad espacial de los parámetros de calidad de agua medidos con los datos satelitales, así como la influencia en el aumento de la eutrofización del lago, lo cual se debe, por un lado, a la estacionalidad, y por otro, a los diferentes usos de suelo tales como la reforestación de pino y eucalipto, actividades agrícolas y áreas urbanas cercanas.

La Cordillera Blanca es una de las áreas más importantes en las montañas de los Andes peruanos, donde los glaciares han experimentado disminuciones. Durán-Alarcón et al. (2015) estudiaron el retroceso de estos glaciares desde 1975 hasta 2010 utilizando imágenes del Mapper Temático (TM) Landsat-5. La extensión del hielo limpio de los glaciares se estimó utilizando los umbrales del Î́ndice de Nieve de Diferencia Normalizada (NDSI). Se aplicó el Índice de Vegetación de Diferencia Normalizada (NDVI) y Temperatura de la Superficie de la Tierra (LST). Las estimaciones del área derivadas de las imágenes del Landsat se compararon con los datos sobre el terreno del glaciar en 1975 y 2010. Los resultados muestran una tendencia decreciente a una velocidad de 4.5 $\mathrm{km}^{2}$ por década de 1975 a 2010, con una pérdida total de $22.5 \mathrm{~km}^{2}$ (58\%).

\section{Aplicación de VANT (Vehículos Aéreos No Tripulados) en cobertura vegetal}

Cruzan et al. (2016), para estimar la distribución y abundancia de tipos de vegetación de 16 hectáreas de Charco Vernal, Estados Unidos, utilizaron un VANT equipado con una cámara integrada (DJI Phantom2), que fue volado a 40 metros de elevación. Concluyeron que los VANT tienen una gran aceptación y precisión para estudios de ecología vegetal, proporcionando numerosas informaciones con mínimo esfuerzo, en comparación con los métodos tradicionales como el transecto o cuadrático y las imágenes satelitales.

Los métodos tradicionales, como mencionan Tay et al. (2018), presentan mayor dificultad para estudios ecológicos y es más difícil cuantificar una población de plantas heterogéneas. Es así como usaron un VANT implementado con tres cámaras digitales (RGB, borde rojo e infrarrojo cercano) a una altura de $50 \mathrm{~m}$ en Schleswig-Holstein, Alemania, para comprobar si estos vehículos reemplazan los métodos actuales en estudios de la dinámica de la población de plantas. Concluyeron que las imágenes obtenidas por el VANT demostraron un elevado potencial para el objeto de estudio, pero el método usado consumió más tiempo del programado debido a los desafíos presentados, tiempo de estudio, tiempo atmosférico y complejidad de manejo del VANT.

La vegetación acuática cumple importantes funciones ecológicas y reguladoras, por lo que es esencial su monitoreo para evaluar los cambios en el ecosistema. Un VANT equipado con una cámara Canon Ixus 70 brindó las imágenes correspondientes que fueron procesadas por GerMap GmbH, y se tuvo como resultado una fácil y eficiente recopilación de información ecológica sobre vegetación acuática no sumergida (Ahmed et al., 2017).

Ivošević et al. (2015), con el objetivo de incluir ecologistas e investigadores en el uso de VANT en la conservación ecológica, desarrollaron una investigación en Corea del Sur (Parque Nacional Chiaksan y Parque Nacional Taeanhaean) usando un VANT Phantom2 visión para el monitoreo de la biodiversidad de especies, a través de imágenes 
de alta resolución y videos incluso en lugares de difícil acceso. Se determinó que los datos e información obtenida son precisos y detallados, y que este equipo puede ser aplicado a diferentes propósitos de investigación.

Asimismo, Ahmed et al. (2017) utilizaron VANT para evaluar cobertura terrestre y mapeo de vegetación. Con ese fin examinaron especies de vegetación en el centro de Ontario-Canadá, utilizando datos de tres cámaras multiespectrales Parrot Sequoia basadas en VANT para analizar cinco tipos de coberturas de suelo (bosque, arbusto, herbáceo, suelo desnudo y edificación). Obtuvieron hasta 95\% de precisión de la clasificación correcta, en comparación con observaciones de campo.

\section{Aplicación de VANT (Vehículos Aéreos No Tripulados) en la dinámica de cuerpos de agua}

El agua es esencial para la supervivencia, por lo que debe ser monitoreada y protegida. En ese sentido, Koparan et al. (2018), en Lamaster Pon, Clemson-Carolina del Sur, desarrollaron un sistema de medición de calidad del agua mediante el uso de un VANT (hexacóptero) equipado con sensores eléctricos que facilitaba la medición in situ de la conductividad eléctrica, oxígeno disuelto y el $\mathrm{pH}$ a una altura de 10 metros. El VANT realizó una exitosa medición de calidad del agua y se demostró que puede ser usado en cuerpos de agua inaccesibles. Su principal limitante fue la corta duración de vuelo.

Miřijovský y Langhammer (2015) analizaron las variaciones en la dinámica fluvial de un arroyo de media montaña de Europa durante los años 2013 y 2014 (época de primavera y de precipitación), empleando un VANT de tipo hexacóptero, una cámara Canon EOS de 500D y un lente especial con una distancia focal de $20 \mathrm{~mm}$. Adquirieron datos fotogramétricos para generar Modelos Digitales de Terreno (MDT) de alta precisión y ortoimágenes con una resolución de $2 \mathrm{~cm}$ por pixel. Con estos determinaron las variaciones del cauce, la erosión a causa del aumento volumétrico y calcularon el área donde se observaron cambios. Finalmente, concluyeron que en la primavera hay más flujos de agua debido al deshielo y, por ende, las inundaciones son mucho mayores.

Teniendo en cuenta que el flujo y la velocidad de un arroyo o de un río son parámetros importantes que deben considerarse para desarrollar planes efectivos de gestión de recursos hídricos, Koutalakis et al. (2019) usaron un VANT para estimar la velocidad superficial del río Aggitis-Grecia. Las imágenes capturadas fueron analizadas por tres tipos de programas (PIVlab, PTVlab y KU-STIV) para la comparación de resultados y para estimar con precisión la velocidad del río, obteniendo datos similares de 0,02 y $3,98 \mathrm{~m} / \mathrm{s} ; 0,12$ y $3,44 \mathrm{~m} / \mathrm{s}$; y 0,04 y $3,99 \mathrm{~m} / \mathrm{s}$ respectivamente. Es necesario complementar este método con una técnica de medidor de corriente de flujo para estudiar la velocidad de la superficie en diferentes alcances, restos vegetales y patrones como ondas superficiales, y tener un resultado mucho más eficiente.

Bandini et al. (2017) realizaron mapas batimétricos de un lago y dos ríos en Dinamarca, de alta calidad, usando un VANT y un sonar sujeto a este, con el objetivo de que la investigación sea modelo para la ingeniería hidráulica y de ciencias hidrológicas. Tuvieron resultados exitosos al medir la batimetría con una precisión de 2,1\% con una profundidad de 30 metros sin verse afectado por la turbidez o material de cama. Con este método se tienen mejores resultados en comparación con las imágenes satelitales, que son deficientes debido a la atenuación de radiación electromagnética en el agua.

Tiria et al. (2018) fueron los primeros en presentar una evaluación global sistemática de las tendencias en las variaciones de vegetación acuática, utilizando un VANT para 155 sitios de estudio en China. Se clasificaron en categorías crecientes y decrecientes, y se obtuvo una acelerada disminución vegetal cuyos causantes principales serían la eutrofización, proliferación de algas, el cultivo de la acuicultura y los cambios climáticos.

\section{Conclusión}

Se determinó que, a partir de la obtención de imágenes satelitales en estudios de pequeña a mediana escala, se puede lograr información de la cobertura boscosa, expansión urbana, índices de vegetación de proporciones estandarizadas, cambio de coberturas vegetales, deforestación, distribución espacial del agua, monitoreo del agua, dinámica lagunar, parámetros de calidad de agua y los umbrales del Índice de Nieve de Diferencia Normalizada. Además, se puede inferir que los VANT (Vehículos Aéreos No Tripulados) en estudios de alta resolución pueden realizar los 
siguientes trabajos en el área ambiental: abundancia vegetal, dinámica de la población de plantas, conservación ecológica, vegetación acuática, mapeo de vegetación, calidad del agua, dinámica fluvial, flujo de ríos, mapas batimétricos de un lago y variaciones vegetales acuáticas.

\section{Literatura citada}

Achícanos, J.A.; Rojas-Robles, R.; Sánchez, J.E.

2018. Análisis y proyección de las coberturas vegetales mediante el uso de sensores remotos y Sistemas de Información Geográfica en la localidad de Suba, BogotáColombia. Gestión y Ambiente, 21(1): 41-58.

Ahmed, O. S.; Shemrock, A.; Chabot, D.; Dillon, C.; Williams, G.; Wasson, R.; Franklin, S. E. 2017. Hierarchical land cover and vegetation classification using multispectral data acquired from an unmanned aerial vehicle. International Journal of Remote Sensing, 38(8-10): 2037-2052.

Álvarez-Rogel, Y.; Conesa, G.C.

2018. Georreferenciación de documentos cartográficos históricos para el análisis del trazado fluvial del Bajo Segura, Vega Media (Murcia, España). GeoFocus, 21: 101-118.

Aliaga, V.S.; Ferrelli, F.; Bohn, V.Y.; Piccolo, M.C.

2016. Utilización de imágenes satelitales para comprender la dinámica lagunar en la Región Pampeana. Revista de Teledeteccion, 46: 133-146.

Bandini, F.; Jakobsen, J.; Olesen, D.; Reyna-Gutiérrez, J. A.; Bauer-Gottwein, P.

2017. Measuring water level in rivers and lakes from lightweight Unmanned Aerial Vehicles. Journal of Hydrology, 548: 237-250.

Briceño, I.; Pérez, W.; San Miguel, D.; Ramos, S.

2018. Determinación de calidad de agua en el lago Vichuquén, con imágenes de satélite Landsat 8, sensor OLI, año 2016, Chile. Revista de teledetección, 52: 67-78.

Cruzan, M.B.; Weinstein, B.G.; Grasty, M.R.; Kohrn, B.F.;

Hendrickson, E.C.; Arredondo, T.M.; Thompson, P.G.

2016. Small unmanned aerial vehicles (Micro-UAVs, drones) in plant ecology. Aplications in Plant Sciences, 4(9): 1600041.

Duffy, J.P.; Cunliffe, A.M.; DeBell, L.; Sandbrook, C.; Wich,

S.A.; Shutler, J.D.; Myers-Smith, I.H.; Varela, M.R.; Anderson, K. 2018. Location, location, location: considerations when using lightweight drones in challenging environments. Remote sensing in ecology and conservation, 4(1): 7-19.

Durán-Alarcón, C.; Gevaert, C.M.; Mattar, C.; Jiménez-Muñoz, C.J.; Pasapera-Gonzales, J.J.; Sobrino, J.A.; Silvia-Vidal, Y.; Fashé-Raymundo, O.; Chávez-Espíritu, T.W.; Santillán-Portilla, N. 2015. Recent trends on glacier area retreat over the group of Nevados Caullaraju-Pastoruri (Cordillera Blanca, Peru) using Landsat imagery. Journal of South American Earth Sciences 59: 19-26.

FAO.

2016. Los bosques y el cambio climático en el Perú. Organización de las Naciones Unidas para la Alimentación y la Agricultura. Roma, Italia. $142 \mathrm{p}$.

Feng, M.; Sexton, J.O.; Channan, S.; Townshend, J.R.

2016. A global, high-resolution (30-m) inland waterbody dataset for 2000: first results of atopographic-spectral classification algorithm. International Journal of Digital Earth, 9(2): 113-133.
Fernández-Lozano, J.; Gutiérrez-Alonso, G.

2016. Aplicaciones geológicas de los drones. Revista de la sociedad geológicas de España, 29(1): 89-105.

Herrera, M.; Porras, L.; Estrella, C.

2018. Propuesta de declaración del espectro electromagnético para Ecuador. Geoespacial, 15(1): 15-32.

Ibarrola-Ulzurrun, E.; Gonzalo-Martin, C.; Marcello-Ruiz, J.;

García-Pedrero, A.; Rodríguez-Esparragon, D.

2017. Fusion of high resolution multispectral imagery in vulnerable coastal and land ecosystems. Sensors 17(2): 228.

Ivošević, B.; Han, Y.; Cho, Y.; Kwon, O.

2015. The use of conservation drones in ecology and wildlife research. Journal of Ecology and Environment, 38(1): 113-118.

Kaplan, G.; Avdan, U.

2017. Object-based water body extraction model using Sentinel-2 satellite imagery. European Journal of Remote Sensing 50(1): 137-143.

Koparan, C.; Bulent, K.A.; Privette, C.V.; Sawyer, C.B.

2018. In situ water quality measurements using an Unmanned Aerial Vehicle (UAV) system. Water, 10: 264.

Koutalakis, P.; Tzoraki, O.; Zaimes, G.

2019. UAV for hydrologic scopes: application of a Low-Cost UAV to estimate surface water velocity by using three different image-based methods. Drones, 3(1): 14.

Lao, R.B.; Peláez, H.D.

2018. Remote sensing and Geographic Information System in the decision making process for land management. Ciencias técnicas agropecuarias, 27(1): 54-65.

Liu, H.; Zhang, Y.; Zhang, X.

2018. Monitoring vegetation coverage in Tongren from 2000 to 2016 based on Landsat7 ETM+ and Landsat8. Anais da Academia Brasileira de Ciências, 90(3): 2721-2730.

Loayza, B.K.J.; Mercedes, C.G.M.; Mestas, V.R.; Quiliche, D.J.P.J.

2017. Estudio del crecimiento urbano y modificación de cobertura vegetal mediante teledetección del Distrito de Végueta. Big Bang Faustiniano, 6(1): 5-8.

Martínez-Barbáchano, R.; Solís-Miranda, G.A.

2018. Caracterización espectral y detección de flecha seca en palma africana en Puntarenas, Costa Rica. Revista Geográfica de América Central, 2(61): 2215-2563.

MINAM, Ministerio del Ambiente.

2017. MINAGRI Y MINAM presentan datos sobre pérdida de cobertura de bosques húmedos amazónicos del 2016. Perú. Disponible en: http://www.minam.gob.pe/ cambioclimatico/2017/09/08/minam-y-minagri-presentarondatos-oficiales-sobre-cobertura-y-perdida-de-bosqueshumedos-amazonicos-al-2016/ (Febrero del 2020).

Miřijovský, J.; Langhammer, J.

2015. Multitemporal monitoring of the morphodynamics of a Mid-Mountain Stream Using UAS photogrammetry. Remote sensing, 7(7): 8586-8609.

Montiel-González, I.; Martínez-Santiago, S.; López S.A.; García H.G. 
2017. Impacto del cambio climático en la agricultura de secano de Aguascalientes, México, para un futuro cercano (20152039). Revista Chapingo Serie Zonas Áridas, 16(1): 1-13.

Paz, P.F.

2018. Estimación de la cobertura aérea de la vegetación herbácea usando sensores remotos. Terra latinoamericana, 36(3): 239-259.

Pino, V.E.

2019. Los drones una herramienta para una agricultura eficiente: un futuro de alta tecnología. IDESIA, 37(1): 75-84.

Salas, R.; Barboza, E.; Beltrán. R.N.; Mamani, J.; Yohel, R.N. 2018. Deforestación en el área de conservación privada Tilacancha: zona de recarga hídrica y de abastecimiento de agua para Chachapoyas. Revista de Investigación Agroproducción Sustentable, 2(3): 54-64.

Tay, J.Y.L.; Erfmeier, A.; Kalwij, J.M.

2018. Reaching new heights: can drones replace current methods to study plant population dynamics? Plant Ecol, 219: 1139-1150.

Tiria, F.L.N.; Bonilla, C.J.S.; Bonilla, C.C.A.

2018. Transformación de las coberturas vegetales y uso del suelo en la llanura amazónica colombiana: el caso de Puerto Leguízamo, Putumayo (Colombia). Cuadernos de Geografía: Revista Colombiana de Geografía, 27 (2): 286-300. 
